\title{
Acknowledgement Correction: Face-to-Face Versus Mobile Versus Blended Weight Loss Program: Randomized Clinical Trial
}

Emalie Hurkmans ${ }^{1,2}, \mathrm{PhD}$; Christophe Matthys ${ }^{3,4,5}, \mathrm{PhD} ;$ An Bogaerts $^{6}, \mathrm{PhD} ;$ Leonie Scheys ${ }^{4,5}$, MSc; Karlien Devloo $^{1}$, MScPT; Jan Seghers ${ }^{1}$, PhD

\footnotetext{
${ }^{1}$ Department of Movement Sciences, University of Leuven, Leuven, Belgium

${ }^{2}$ Department of Social Affairs and Health, Ecorys, Rotterdam, Netherlands

${ }^{3}$ Department of Endocrinology, University Hospitals Leuven, Leuven, Belgium

${ }^{4}$ Department of Chronic Diseases, Metabolism and Ageing, University of Leuven, Leuven, Belgium

${ }^{5}$ Department of Clinical and Experimental Endocrinology, University of Leuven, Leuven, Belgium

${ }^{6}$ Faculty of Movement and Rehabilitation Sciences, University of Leuven, Leuven, Belgium
}

\section{Corresponding Author:}

Jan Seghers, PhD

Department of Movement Sciences

University of Leuven

Tervuursevest 101

Leuven,

Belgium

Phone: 3216329048

Email: jan.seghers@kuleuven.be

\section{Related Article:}

Correction of: http://mhealth.jmir.org/2018/1/e14/

(JMIR Mhealth Uhealth 2018;6(3):e10159) doi: 10.2196/10159

The authors of "Face-to-Face Versus Mobile Versus Blended Weight Loss Program: Randomized Clinical Trial" (JMIR mHealth uHealth 2018;6(1):e14) would like to change the Acknowledgments section of their paper to the following:

This project is partially funded and realized in collaboration with imec, Belgium. BrandNewHealth developed the weight loss app.
The corrected article will appear in the online version of the paper on the JMIR website on March 15, 2018, together with the publication of this correction notice. Because this was made after submission to PubMed or Pubmed Central and other full-text repositories, the corrected article also has been re-submitted to those repositories.

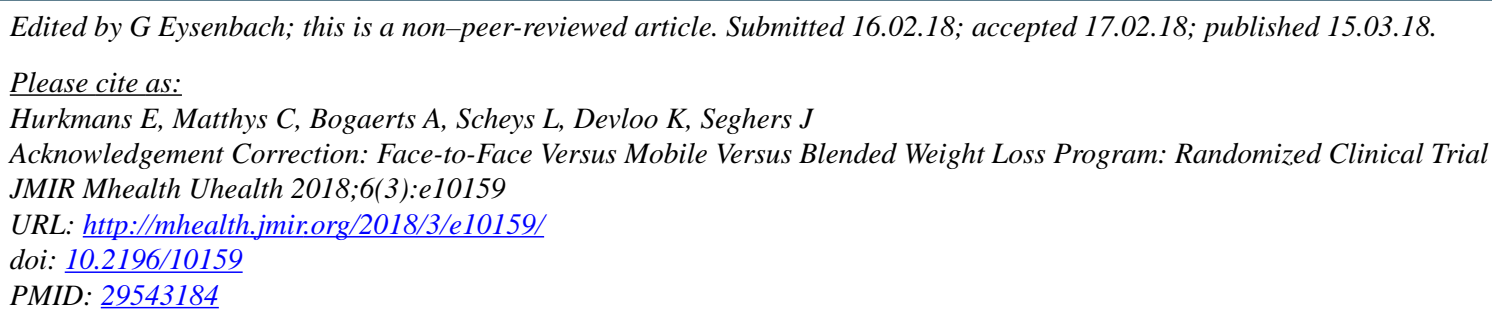

CEmalie Hurkmans, Christophe Matthys, An Bogaerts, Leonie Scheys, Karlien Devloo, Jan Seghers. Originally published in JMIR Mhealth and Uhealth (http://mhealth.jmir.org), 15.03.2018. This is an open-access article distributed under the terms of the Creative Commons Attribution License (https://creativecommons.org/licenses/by/4.0/), which permits unrestricted use, distribution, and reproduction in any medium, provided the original work, first published in JMIR mhealth and uhealth, is properly cited. The complete bibliographic information, a link to the original publication on http://mhealth.jmir.org/, as well as this copyright and license information must be included. 